\title{
On the distillation of mercury
}

\section{M.M.E. Millon}

To cite this article: M.M.E. Millon (1847) On the distillation of mercury, Philosophical Magazine Series 3, 30:199, 144-146, DOI: 10.1080/14786444708645672

To link to this article: http://dx.doi.org/10.1080/14786444708645672

曲 Published online: 30 Apr 2009.

Submit your article to this journal $2 \pi$

Џ Article views: 3

Q View related articles $₫$ 
by the fire of the brass wire still hot, there is produced exactly the heat necessary to continue it incandescent. If the spirals of a brass wire approach each other without touching, the vapour of the alcohol oxidizes, and can act on a large surface; the heat is so much increased by it, that the spirals remain red-hot; if the spirals be further removed from each other, ignition ceases, the heat produced by the imperfect combustion not being sufficient to heat the spirals, which before almost touched.

As to what relates to spongy platina, the globules are surrounded with an atmosphere of hydrogen, oxidation takes place at the same time, heat is consequently developed by the large surface of the spongy platina, and a reiterated contact of the gases so increases the heat, that the gas is inflamed. Platina would not possess this specific quality if it were not so refractory as not to fuse by the heat of inflamed hydrogen.

M. Reinsch has also found that this property belongs to metals which fuse readily and are not capable of being made into wire. He made the following experiment: some asbestos was moistened with a solution of these metals, and after having made it red-hot, and it had become brittle by the ignition, it was moistened with a little alcohol, after which it was cautiously rolled round the wick; the metallized asbestos was kept for some time red-hot in the flame of the alcohol, a glass tube being held over it: asbestos which had been prepared with chloride of platina remained vividly red-hot. Iron answers best for this experiment : asbestos moistened with a solution of sulphate of iron and then immersed in solution of ammonia, and washed in water and dried quickly, remains red like an iron wire. Asbestos moistened with a solution of gold and heated to redness, remains red-hot in the vapour of alcohol like a platina wire. 'The same occurs when asbestos is moistened with a solution of silver. It has been above stated that gold and silver wires possess the property of ignition but imperfectly; which shows that they are subject to soften readily. A wire prepared with chloride of tin and oxalate of ammonia, remains only a few seconds red, even under the glass tube, the tin being too suddenly converted into oxide. The case is nearly the same with lead, but it remains red rather longer. Cobalt and nickel possess the property of igniting like iron. Manganese and chromium remain red for a very short time. Arsenic is suddenly volatilized, the wire however remains red for a few seconds; the same occurs with mercury. Bismuth possesses this property of ignition in a rather less degree than iron. Cadmium resembles tin. Zinc is the only metal which does not at all ignite.

The author is of opinion that platinized asbestos may become an important substance for the condensation of several gases, as also for the preparation of vinegar.-Journ. de Pharm. et de Ch., Nov. 1846.

\section{ON THE DISTILIATION OF MERCURY. BY M. M. E. MILLON.}

When mercury is distilled, it is observable that the volatilization takes place more slowly at the period in which the last portions of 
the metal pass into the receiver. If the mercury distilled at the commencement of the operation be kept separate from that obtained at the end of it, it is easily proved that these two portions are of unequal volatility.

Fifty kilogrammes of mercury were distilled, and the first and last kilogramme distilled were kept separate; both of them were redistilled and then submitted to the following experiment: four small and similar retorts were selected, each of which when half-filled was capable of holding 100 grammes of mercury. Each of the retorts charged with 100 grammes of the metal was immersed into an alloybath in fusion; and the heat was raised till the mercury in all of them boiled. The mercury distilled condensed in the neck, and was collected and weighed. The four retorts did not yield equal quantities; but on putting aside those which differed the most, it was easy to select two, which, subjected to the above proof, furnished nearly an equal quantity of mercury. Thus in three parallel operations with these two retorts distinguished by the letters $A$ and $B-$

In the same time, in the same bath.

Retort A. First operation. Mercury distilled $48 \cdot 5$ grammes.

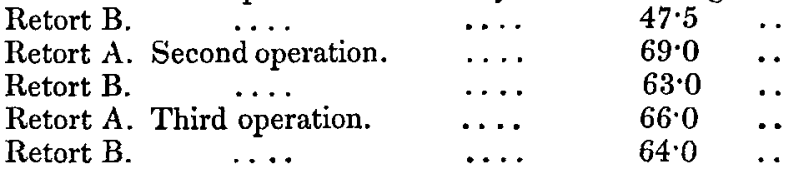

It is to be remarked that in these three operations the retort $A$. always yielded a little more than the retort $B$. This circumstance was attended to in the experiments which follow.

'The following are the differences obtained by submitting to comparative trial in the alloy-bath, mercury taken from the first and last kilogramme obtained from the distillation of 50 kilogrammes of the metal.

The retort A., which yielded the most, received the mercury collected at the close of the distillation; the retort $B$. that obtained at the commencement.

Mercury distilled during the same time in the same bath.

Retort A. First operation. Containing $100 \mathrm{grms}$. of metal $19 \cdot 0 \mathrm{grms}$. $\begin{array}{llll}\text { Retort B. } & \ldots . & \ldots \ldots & 49.0 \ldots \\ \text { Retort A. Second operation. } & \ldots . & \ldots . & 15.7\end{array}$

Care must be taken, in these comparative distillations, to moderate the temperature of the alloy-bath as soon as the mercury begins to spot the dome of the retort.

Mercury thus affected in a marked manner in its mode of distillation would appear to possess some difference in its degree of purity; but the author attempted in vain to discover any difference by reagents; the mercury of the first and last kilogramme was similarly affected in all the examinations to which it was submitted.

It then occurred to the author to try whether the addition of any foreign metal, in quantity so small as to escape analysis, could affect the volatility of the mercury. 'The results were of considerable in. 
terest; the thousandth or even ten-thousandth of a foreign metal is sufficient to cause very characteristic differences in the resulta of parallel experiments.

A ten-thousandth of lead added to mercury stops its distillation almost entirely. In the following comparative experiments, the mercury which distilled with the greatest difficulty was put into the retort $A$., which yielded most quickly.

First operation.-Retort A., containing 100 grammes of mercury, to which 1-10,000dth of lead had been added, yielded in a given time by distillation in the bath 5 grammes of mercury.

Retort B., containing the same quantity of mercury without any lead, heated for the same time in the same bath, gave by distillation 67 grammes of mercury.

Second operation.- Retort A., containing 100 grammes of mercury, to which 1 -1000dth of lead had been added, yielded in a given time by distillation in the bath 2 grammes of mercury.

Retort B., containing the same quantity of mercury without any lead, heated for the same time in the same bath, gave by distillation $\mathbf{5 5}$ grammes of mercury.

Zinc was substituted for lead, and when in the proportion of $1-10,000 \mathrm{dth}$, the mercury distilled was to that obtained without the zinc as 6.5 grms. to 72 .

Some metals have no sensible influence on the distillation of mercury : among these are gold, iridium, silver, copper, tin, nickel, cadmium and arsenic. The action of platina is precisely the reverse of that of lead and zinc : it accelerates distillation, but less than lead and zinc retard it; in order that platina may produce this effect, it must be digested in the mercury for a day or two at the temperature of $122^{\circ}$ to $176^{\circ}$ F.-Ann. de Ch. et de Phys., Novembre 1846.

\section{EASY METHOD OF PREPARING IODIDE OF POTASSIUM.}

M. Pypers subjects to a moderate heat a mixture of 100 parts of iodine, 75 of carbonate of potash, 30 of iron filings and 120 of water. 'The mass is to be dried, and then heated to redness; the resulting reddish powder is to be treated with water, and the solution obtained filtered and evaporated to dryness. One hundred parts of iodine yield 135 of very white, but slightly alkaline iodide of potassium.Journ. de Chim. Méd., Decembre 1846.

\section{FORMATION OF GLYCERINE. BY M. ROCHLEDER.}

If castor-oil be dissolved in absolute alcuhol and a current of dry hydrochloric acid gas be directed into the heated solution, the oil undergoes decomposition. When the liquor is agitated with water, after the hydrochloric gas has acted for a sufficient time, an emulsion is obtained, which gradually separates into two portions, one of which is oily and floats, the other is watery and extremely acid; the latter is to be poured off and evaporated by a water-bath. At first hydrochloric acid is disengaged, and there remains a syrupy and yellowish mass 